\title{
Shunting in cryptococcal meningitis
}

\author{
Jacob Cherian, MD, ${ }^{1}$ Robert L. Atmar, MD, ${ }^{2}$ and Shankar P. Gopinath, MD1 \\ Departments of ${ }^{1}$ Neurosurgery and ${ }^{2}$ Medicine, Section of Infectious Diseases, Baylor College of Medicine, Houston, Texas
}

OBJECTIVE Patients with cryptococcal meningitis often develop symptomatic intracranial hypertension. The need for permanent CSF diversion in these cases remains unclear.

METHODS Cases of cryptococcal meningitis over a 5-year period were reviewed from a single, large teaching hospital. Sources of identification included ICD-9 codes, operative logs, and microscopy laboratory records.

RESULTS Fifty cases of cryptococcal meningitis were identified. Ninety-eight percent (49/50) of patients were HIV positive. Opening pressure on initial lumbar puncture diagnosing cryptococcal meningitis was elevated $\left(>25 \mathrm{~cm} \mathrm{H}_{2} \mathrm{O}\right)$ in 33 cases and normal $\left(\leq 25 \mathrm{~cm} \mathrm{H}_{2} \mathrm{O}\right)$ in 17 cases. Thirty-eight patients ultimately developed elevated opening pressure over a follow-up period ranging from weeks to years.

Serial lumbar punctures for relief of intracranial hypertension were performed in 29 cases. Thirteen of these patients ultimately had shunting procedures performed after failing to improve clinically. Two factors were significantly associated with the need for shunting: patients undergoing shunt placement were more likely to be women $(5 / 13$ vs $0 / 16 ; p=0.01)$ and to have a pattern of increasing CSF cryptococcal antigen (10/13 vs $3 / 16$ cases; $p=0.003$ ). All patients re-presenting with mycological relapse either underwent or were offered shunt placement.

CONCLUSIONS Neurosurgeons are often asked to consider CSF diversion in cases of cryptococcal meningitis complicated by intracranial hypertension. Most patients do well with serial lumbar punctures combined with antifungal therapy. When required, shunting generally provided sustained relief from intracranial hypertension symptoms. Ventriculoperitoneal shunts are the favored method of diversion. To the authors' knowledge, the present study is the largest series on diversionary shunts in primarily HIV-positive patients with this problem.

http://thejns.org/doi/abs/10.3171/2015.4.JNS15255

KEY WORDS cryptococcal meningitis; shunt; lumbar puncture; headache; infection

$\mathrm{C}$ RYPTOCOCCAL meningitis is a major opportunistic infection seen commonly in the immunocompromised, particularly in those infected with HIV. ${ }^{26}$ Elevated CSF pressures are often seen in these patients. Significant neurological decline can occur if elevated CSF pressures are inadequately managed. ${ }^{28,29,33,39}$ Various treatment strategies to address intracranial hypertension in this setting have been described, including medical management, serial lumbar punctures, external lumbar and ventricular drain placement, and either ventricular or lumbar shunting..$^{26}$ The literature remains unclear as to when each approach is indicated and how often patients require neurosurgical intervention. The present study offers a retrospective review of our experience at a single institution over a 5-year period.

\section{Methods}

A retrospective electronic chart review was conducted of records from a single large teaching hospital of 586 beds serving a major metropolitan area. Using ICD-9 codes, patient admission and discharge diagnoses of cryptococcal meningitis between September 2008 and February 2013 were identified. Lists of positive CSF cultures and cryptococcal antigens were also obtained for this time period to identify infected persons. Individual patient encounters were then inspected for pertinent data regarding diagnosis, management pattern, and outcome. The review was approved by the Baylor College of Medicine institutional review board.

The open-source R software package (http://www. rproject.org/) was used to calculate 2-sided Fisher's exact test $p$ values to analyze contingency tables of comparison groups, Welch's variant 2-sample t-test $\mathrm{p}$ values for comparison of distribution means, Wilcoxon rank-sum $\mathrm{p}$ values for comparison of distribution medians of cryptococcal antigen levels, and Pearson correlation coefficients for regression analyses. For analysis of statistical significance, a $\mathrm{p}$ value threshold $<0.05$ was used. 


\section{Results}

Fifty cases of cryptococcal meningitis were identified, some of which were initially diagnosed prior to the study period. The average age was 39 years (range 22-57 years), and with the exception of a single case, study patients (49/50) were HIV positive. The patient without HIV infection was infected with Cryptococcus gattii; the rest of the patients were infected with C. neoformans. Eightysix percent (43/50) of patients had confirmation by positive CSF culture, $10 \%(5 / 50)$ had only positive CSF cryptococcal antigen tests, and 4\% (2/50) were diagnosed at outside facilities with diagnosis records unavailable for retrospective review.

The 4 most common presenting symptoms included headache in $72 \%(36 / 50)$ of patients, nausea and vomiting in $42 \%(21 / 50)$, altered mental status in $26 \%(13 / 50)$, and meningismus in 20\% (10/50). Quantitative cryptococcal antigen titer level from CSF on initial lumbar puncture diagnosing cryptococcal meningitis ranged from 1:32 to $1: 65,536$ (normal is nonreactive). Quantitative cryptococcal antigen level adjusted logarithmically did not correlate with opening pressure $(r=0.16, p=0.28)$. Table 1 summarizes patient demographic data.

The Infectious Diseases Service was consulted in all cases. Patients received dual induction therapy with flucytosine in combination with either amphotericin B deoxycholate (Fungizone) or liposomal amphotericin B (AmBisome) in 90\% (45/50) of cases. Table 2 summarizes antifungal management. Among the 5 patients who did not receive standard induction therapy, there were 3 cases of early discharge due to death or discharge against medical advice. Fluconazole was used alone in another case because of severe renal failure and what was believed to be very mild cryptococcal meningitis. It is unclear why 5-flucytosine was not given with amphotericin B in the remaining case.

When feasible, CSF cultures were taken on diagnosis, 2 weeks later, and during additional lumbar punctures per-

TABLE 1. Summary of demographic and presentation data in $\mathbf{5 0}$ patients with cryptococcal meningitis

\begin{tabular}{lcc}
\hline Variable & No. of Pts $(\mathrm{n}=50)$ & $\%$ \\
\hline Mean age, yrs & 39 & NA \\
\hline Sex & & \\
\hline M & 43 & 86 \\
\hline F & 7 & \\
\hline HIV status & 49 & 98 \\
\hline Positive & 1 & 2 \\
\hline Negative & & 72 \\
\hline Presentation & 36 & 42 \\
\hline Headache & 21 & 12 \\
\hline Nausea, vomiting & 6 & 26 \\
\hline Seizure & 13 & 20 \\
\hline AMS & 10 & 8 \\
\hline Meningismus & 4 &
\end{tabular}

AMS = altered mental status; NA = not applicable; Pts = patients. formed for therapeutic CSF drainage. Lumbar puncture opening pressures were measured with the patient in the lateral decubitus position with hips and knees extended. Ten percent $(5 / 50)$ of patients had culture-confirmed mycological relapse. In all 5 cases, relapse was attributed to a history of medication noncompliance. In an additional 9 cases, relapse was suspected on clinical grounds without positive fungal cultures. Among these 9 cases, 3 patients had a history of medication noncompliance and 4 were ultimately believed to have immune reconstitution inflammatory syndrome (IRIS) due to highly active antiretroviral therapy (HAART).

All 50 patients underwent noncontrast CT head imaging. Per the subjective read of the neuroradiologist, only 12 persons demonstrated ventriculomegaly. In 1 patient, there was obstructive hydrocephalus secondary to severe cerebral swelling around the fourth ventricle. Ten of the persons with ventriculomegaly also had concomitant intracranial hypertension. The majority $(28 / 38,74 \%)$ of patients who developed elevated opening pressures had no changes in ventricular morphology. The presence of ventriculomegaly as determined by the neuroradiologist did not significantly correlate with altered mental status presentation.

On review, examination for papilledema was not consistently documented. In those with intracranial hypertension, 5 patients had documented papilledema and 4 of these 5 had opening pressures exceeding $47 \mathrm{~cm} \mathrm{H}_{2} \mathrm{O}$.

Altogether, $42 \%(21 / 50)$ of cases were treated exclusively with medical management centered on antifungal therapy, $32 \%(16 / 50)$ were treated with medical management plus serial lumbar punctures, and 26\% (13/50) had neurosurgical management with ventriculoperitoneal or lumboperitoneal shunts. Figures 1 and 2 present flow charts categorizing interventions and outcomes.

\section{Opening Pressures and Therapeutic Drainage}

Opening pressure on initial lumbar puncture diagnosing cryptococcal meningitis was elevated $\left(>25 \mathrm{~cm} \mathrm{H}_{2} \mathrm{O}\right)$ in 33 cases and normal $\left(\leq 25 \mathrm{~cm} \mathrm{H}_{2} \mathrm{O}\right)$ in 17 cases. Approximately $30 \%(5 / 17)$ of patients with initially normal opening pressures later had elevated opening pressures on subsequent lumbar punctures over a period of weeks to years.

TABLE 2. Summary of antifungal and intracranial hypertension management

\begin{tabular}{|c|c|c|}
\hline Type of Management & No. of Pts & $\%$ \\
\hline \multicolumn{3}{|l|}{ Antifungal* $^{*}$} \\
\hline Fungizone, AmBisome, flucytosine, fluconazole & 28 & 56 \\
\hline Fungizone, flucytosine, fluconazole & 16 & 32 \\
\hline AmBisome, flucytosine, fluconazole & 1 & 2 \\
\hline Other & 5 & 10 \\
\hline \multicolumn{3}{|l|}{ Intracranial hypertension } \\
\hline Medical only & 21 & 42 \\
\hline Serial LPs & 16 & 32 \\
\hline Shunt & 13 & 26 \\
\hline
\end{tabular}




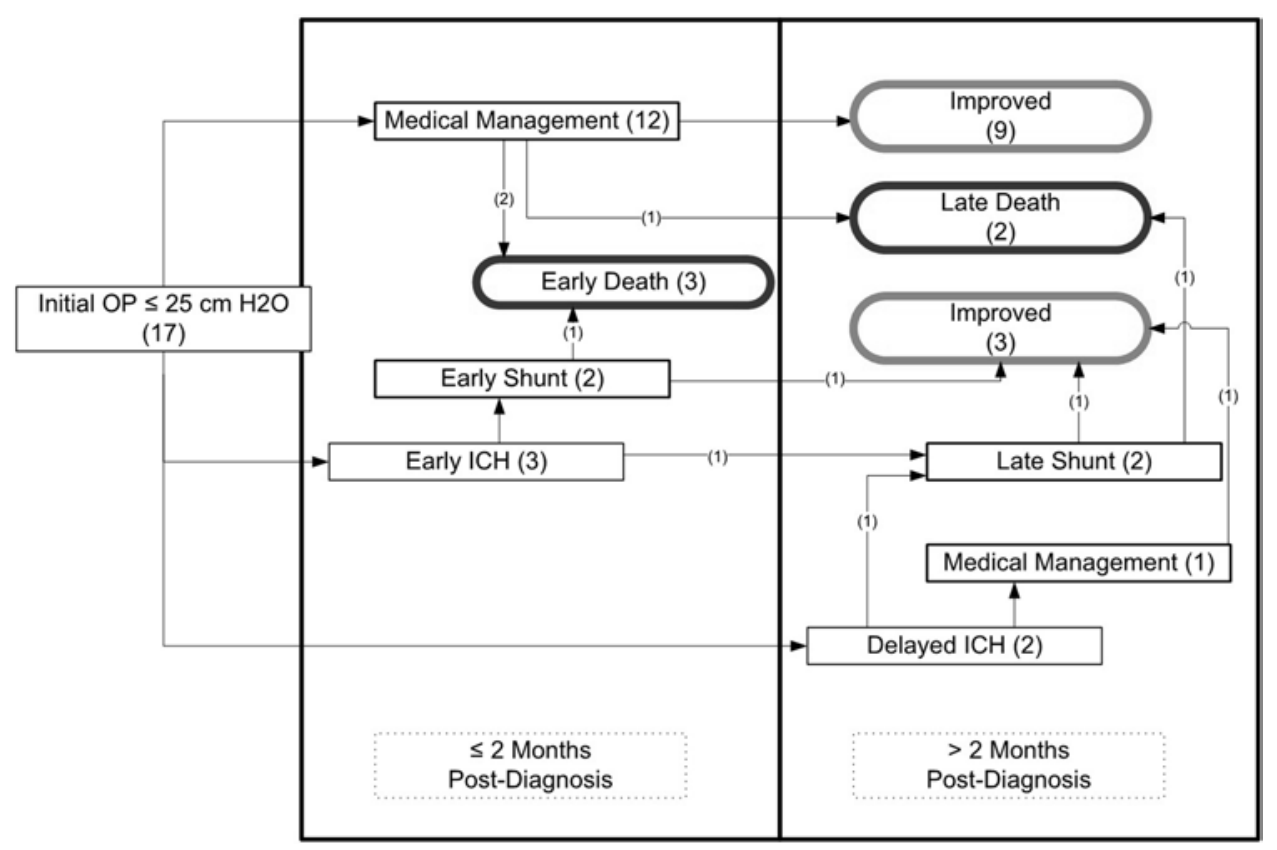

FIG. 1. Flow chart summarizing management and outcomes in patients diagnosed with cryptococcal meningitis who initially presented with normal opening pressures. All patients who received shunts were managed preoperatively with serial therapeutic lumbar punctures. One patient with delayed intracranial hypertension received a shunt within 1 month of diagnosis of intracranial hypertension. $\mathrm{ICH}=$ intracranial hypertension; $\mathrm{OP}=$ opening pressure on lumbar puncture.

The remaining 12 patients with normal opening pressures on initial and subsequent lumbar punctures were treated with antifungal management only. Nine patients with elevated opening pressures were also managed medically, without serial lumbar punctures or surgical interventions. In most of these latter cases, opening pressures were only marginally high and symptoms of headache were less pronounced.

Acetazolamide and mannitol were not used for medical management of intracranial hypertension. Corticosteroids, primarily prednisone, were used in the setting of IRIS, which was ultimately diagnosed in $28 \%(14 / 50)$ of

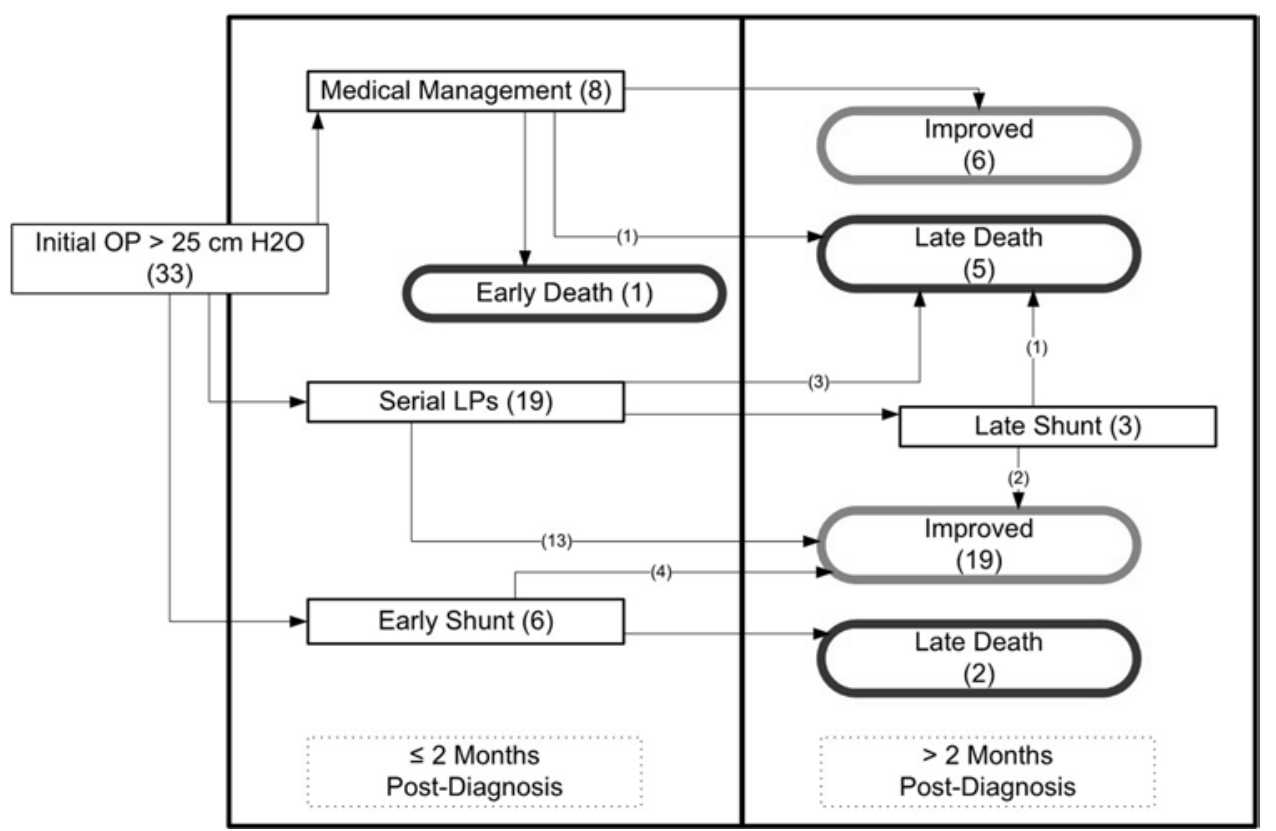

FIG. 2. Flow chart summarizing management and outcomes in patients diagnosed with cryptococcal meningitis who initially presented with elevated opening pressures. All patients who received shunts were managed preoperatively with serial therapeutic lumbar punctures. 
patients. This usually presented with relapsed symptoms of headache, nausea, and vomiting, as well as elevated opening pressures weeks to months after initiation or reinitiation of HAART.

Serial lumbar punctures for relief of intracranial hypertension were performed in 29 cases. Drainage was performed with the goal of reducing closing pressure to less than $50 \%$ of opening pressure or to less than $25 \mathrm{~cm}$ $\mathrm{H}_{2} \mathrm{O}$, whichever was higher. Thirteen of these patients ultimately required ventriculoperitoneal or lumboperitoneal shunt placement. In the 16 cases managed with serial lumbar punctures alone, 14 patients had a good response to therapeutic serial lumbar puncture drainage and were discharged to home. The remaining 2 patients were discharged to hospice.

\section{Shunt Placement}

Neurosurgery consult was sought in $44 \%$ (22/50) of cases, with nearly $60 \%$ (13/22) of these patients ultimately undergoing either ventriculoperitoneal or lumboperitoneal shunt placement. Shunts were considered when patients did not obtain adequate relief of headache after lumbar puncture drainage, had persistently elevated opening pressures after at least 3 taps, or could no longer tolerate further lumbar punctures. In the 9 patients who were seen by neurosurgery but not treated with shunt placement, 3 patients did not have intracranial hypertension and $5 \mathrm{im}-$ proved with serial lumbar punctures.

Table 3 describes characteristics of patients who underwent shunt placement. There was a median of 7 lumbar punctures performed prior to shunt placement (range 2-32). This was not statistically different than the number of lumbar punctures recorded for patients undergoing serial lumbar punctures alone. Reviewed lumbar puncture counts were based primarily on laboratory records of collected CSF samples. This undercounts lumbar punctures performed for therapeutic nondiagnostic drainage. One patient had at least 32 lumbar punctures performed prior to shunt placement. Most of these taps were performed at an outside facility prior to transfer to our institution for shunt insertion.

The time from diagnosis of intracranial hypertension to date of shunt placement was a median of 27 days. Nine of 13 shunts were placed within 2 months of diagnosis of intracranial hypertension. In patients who received shunts more than 2 months after development of intracranial hypertension, 2 patients underwent shunt insertion during periods of mycological relapse secondary to medication noncompliance and 1 patient had suspected IRIS after reinitiating HAART.

In patients who received shunts, fixed medium-pressure valve ventriculoperitoneal shunts were placed in $69 \%$ $(9 / 13)$ of patients and lumboperitoneal shunts in the remainder. In all but 1 case, symptoms of intracranial hypertension improved with shunt placement. Fifty-four percent $(7 / 13)$ of patients were discharged and remain alive with clinical follow-up of more than 1 year. Two of 13 patients who had shunt insertion required repeat surgery for shunt revision. The first developed a subdural hematoma secondary to overdrainage and required high-pressure valve substitution. The second was found to have a malfunction- ing shunt during a readmission for mycological relapse. This shunt was successfully explanted.

\section{Patients Receiving Serial Lumbar Punctures Versus Shunt Placement}

Patients who underwent serial lumbar punctures and then had shunts placed were compared with patients receiving serial lumbar punctures only (Table 4). Two factors were more common in patients who underwent a shunt procedure. Patients who received shunts were more likely to be women $(5 / 13$ vs $0 / 16 ; p=0.01)$. Additionally, patients who underwent a shunt procedure were significantly more likely to have an increased CSF cryptococcal antigen level after diagnostic lumbar puncture (10/13 vs $3 / 16$ cases, $p=0.003$ ).

\section{Mortality}

There were 13 deaths during the study period and all occurred among HIV-infected patients. Eight deaths were secondary to concomitant infection related to immunodeficiency or after changes in code status due to respiratory failure or progression of terminal AIDS. Two patients with opening pressures in the upper normal range were given comfort care only at the request of their families after their altered mental status failed to improve. Two deaths were attributed directly to disseminated cryptococcal infection, with 1 of these 2 patients refusing an offer of a shunt for treatment of intracranial hypertension. The remaining death occurred in the outpatient setting for unknown causes 4 months after discharge diagnosis of cryptococcal meningitis.

\section{Discussion}

Cryptococcosis is a major opportunistic pathogen worldwide, and is particularly common in the immunocompromised HIV-positive population. Although the widespread use of HAART has lowered the incidence of cryptococcosis, the incidence and mortality of this infection remain high in areas with uncontrolled HIV disease and among patients with limited access to HAART. ${ }^{26}$

Intracranial hypertension is well reported in both HIVpositive and -negative patients who develop cryptococcal meningitis. ${ }^{8}, 13$ Symptoms include headache, nausea, vomiting, and altered mental status. In a large series of 221 patients, Graybill et al..$^{13}$ demonstrated that more than $50 \%$ (119/221) of patients had opening pressures $\geq 25 \mathrm{~cm} \mathrm{H}_{2} \mathrm{O}$ on initial lumbar puncture. Failure to address the consequences of intracranial hypertension can be significantly detrimental. . $, 8,11,24$ Current guidelines strongly emphasize measuring opening pressure on initial diagnostic lumbar puncture, and performing serial lumbar puncture drainage for pressure and symptom control. ${ }^{26}$ Papilledema, when documented, was associated with high opening pressures in this series, consistent with prior work by Graybill et al. ${ }^{13}$

Neurosurgeons are often asked to consider permanent or temporary methods of diversion in these cases. Despite elevated opening pressures, many of these patients do not demonstrate ventriculomegaly by imaging. ${ }^{13,26,33,34,36}$ In this series, 28 of 38 patients with elevated opening pressures had no changes in ventricular morphology. 


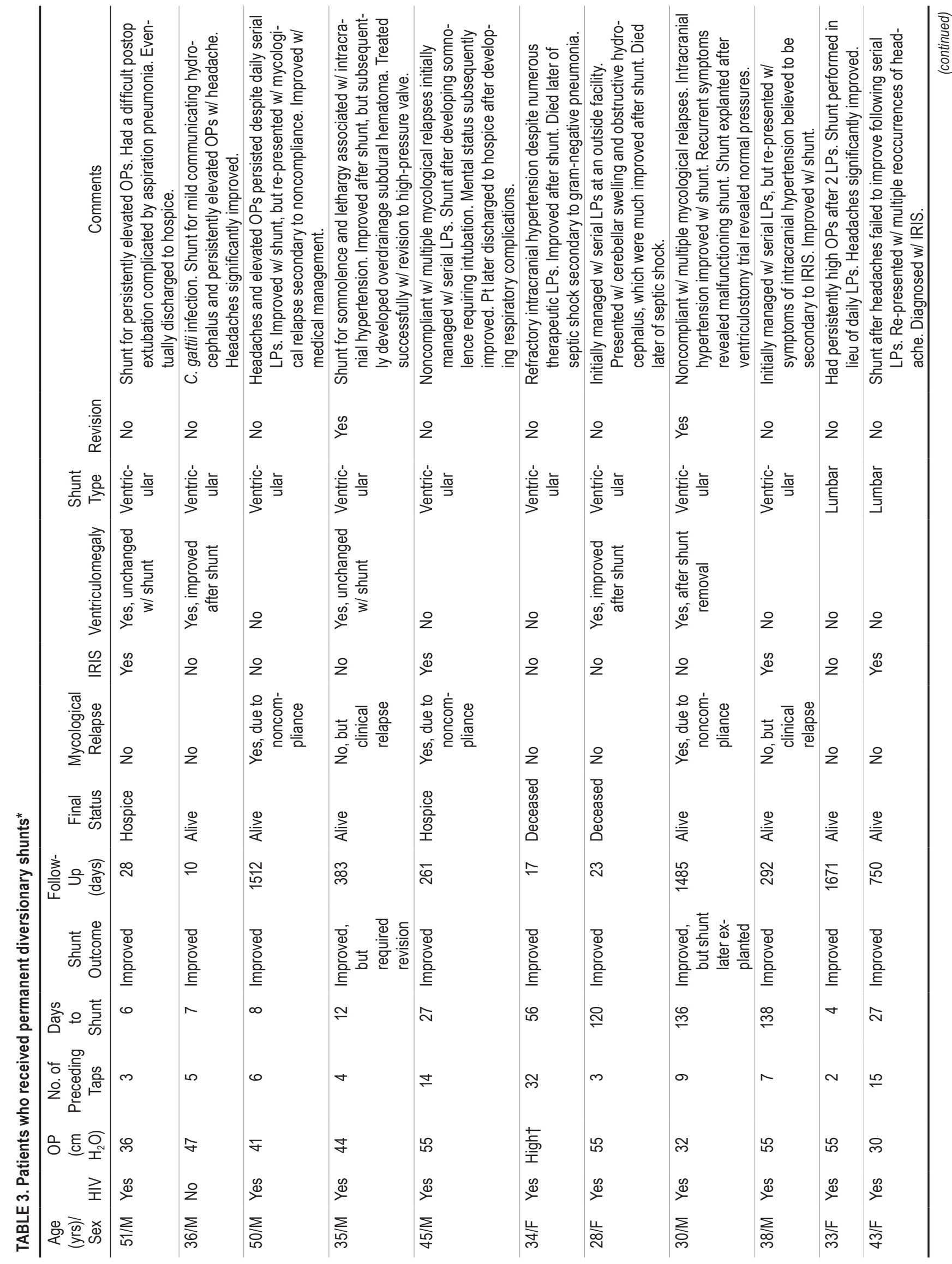




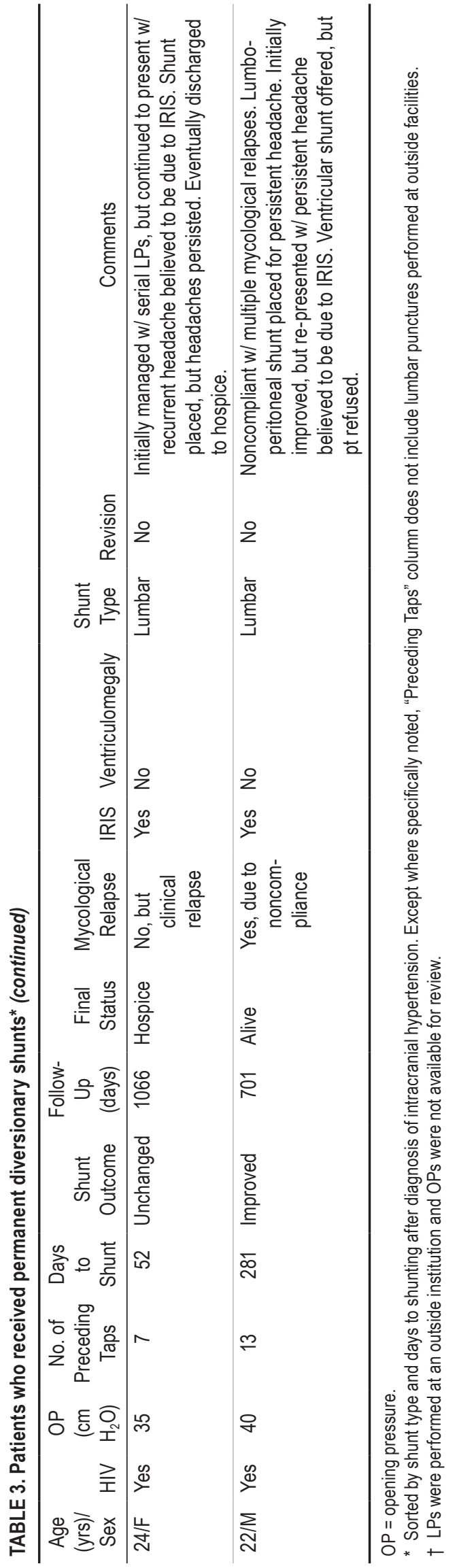

\section{Treating Elevated Pressures}

Various treatment strategies to address elevated intracranial pressure in the management of cryptococcal meningitis have been proposed. Medical management alone using acetazolamide and steroids is not helpful..$^{13,22}$ In HIV-negative patients, ventriculoperitoneal shunting is well described by multiple authors and has had excellent results. ${ }^{6,10,11,15,19,25,27,28,33,35-37}$ In HIV-positive patients, however, the use of shunting to treat intracranial hypertension has been surprisingly sparse. Rather, there has been a greater emphasis on the use of serial lumbar punctures and lumbar drains for therapeutic relief of CSF pressure. Table 5 summarizes selected published data on HIV-infected patients with cryptococcal meningitis who received shunts.

In 1998, for example, Fessler et al. ${ }^{12}$ presented a prospective series identifying $10 \mathrm{HIV}$-positive patients with cryptococcal meningitis and elevated opening pressures $\geq 20 \mathrm{~cm} \mathrm{H}_{2} \mathrm{O}$ on diagnostic lumbar punctures. All had symptoms of intracranial hypertension. Nine patients underwent a short trial of serial lumbar punctures for up to 72 hours and as frequently as twice daily, but all failed to demonstrate sustained relief from symptoms of headache, nausea, vomiting, and altered mental status. All 10 patients subsequently underwent lumbar drainage via an external lumbar drain. Although all 10 patients had significant relief in symptoms, 8 patients could not be weaned off external lumbar drainage without recurrence of symptoms. Lumboperitoneal shunts were then placed in these patients, with good response.

More recently, a large retrospective analysis by Bicanic et al. ${ }^{4}$ in 2009 demonstrated significant value of multiple scheduled lumbar punctures performed within the first 2 weeks of cryptococcal meningitis treatment. Elevated opening pressures were treated with therapeutic drainage of up to $30 \mathrm{ml}$ of CSF to achieve a closing pressure below $20 \mathrm{~cm} \mathrm{H}_{2} \mathrm{O}$, or less than $50 \%$ of opening pressure. Under this protocol, there were no major differences in outcome based on initial opening pressures. With 10 weeks of follow-up, only 4 patients required therapeutic lumbar puncture drainage after Day 14. One patient ultimately required 5 days of drainage via lumbar drain. No shunts were placed.

This is the largest series in which permanent diversionary shunts were placed for the management of intracranial hypertension secondary to cryptococcal meningitis in primarily HIV-positive patients. This higher rate of shunting may be partly attributed to a high rate of clinical and mycological relapse of $28 \%$ (14/50) of patients and may reflect the medication noncompliance of our subpopulation. Fifty percent (7/14) of these patients with relapsed symptoms of intracranial hypertension ultimately received shunts. Of the 5 patients presenting with culture-confirmed mycological relapse, permanent shunts were implanted in 4 and offered to the fifth patient, who was ultimately given comfort care at the request of the family.

\section{Shunt Placement in the Immunocompromised}

As described by Woodworth et al., ${ }^{40}$ there may be multiple conjectural reasons for hesitation regarding shunt placement in HIV-infected patients. First, implanted shunts might be more susceptible to infection in the immu- 
TABLE 4. Patients with intracranial hypertension who had either serial lumbar punctures alone or serial lumbar puncture followed by shunt placement

\begin{tabular}{|c|c|c|c|}
\hline Variable & Serial LP $(n=16)$ & Shunt $(n=13)$ & $p$ Value \\
\hline No. of male pts (\%) & $16(100)$ & $8(62)$ & 0.01 \\
\hline Mean age, yrs (SD) & $42(8.8)$ & $36(9.2)$ & 0.07 \\
\hline Mean no. of LPs (SD) & $5.4(2.0)$ & $9.2(8.1)$ & 0.12 \\
\hline \multicolumn{4}{|l|}{ At diagnosis } \\
\hline Mean OP (SD) & $42(10.1)$ & 37 (14.1) & 0.28 \\
\hline Median CSF cryptococcal Ag & 4096 & 4096 & 0.72 \\
\hline Mean CSF protein (SD) & $88.3(57.7)$ & $59.8(35.0)$ & 0.12 \\
\hline \multicolumn{4}{|l|}{ At time of elevated ICP } \\
\hline Mean OP (SD) & $42(9.5)$ & $44(9.5)$ & 0.55 \\
\hline Median CSF cryptococcal Ag & 4096 & 2048 & 0.33 \\
\hline Mean CSF protein (SD) & $88.3(57.7)$ & $59.6(35.6)$ & 0.12 \\
\hline Documented papilledema (\%) & $2(13)$ & $3(23)$ & 0.63 \\
\hline Ventriculomegaly (\%) & $2(13)$ & $5(38)$ & 0.19 \\
\hline \multicolumn{4}{|l|}{ At time of intervention } \\
\hline Culture-confirmed mycological relapse (\%) & $0(0)$ & $3(23)$ & 0.08 \\
\hline Clinical relapse or IRIS (\%) & $6(38)$ & $3(23)$ & 0.45 \\
\hline \multicolumn{4}{|l|}{ Cryptococcal Ag pattern } \\
\hline Median CSF cryptococcal Ag, maximum & 4096 & 8192 & 0.37 \\
\hline Increasing CSF cryptococcal Ag (\%)* & $3(19)$ & $10(77)$ & $<0.01$ \\
\hline 4-fold rise in CSF cryptococcal Ag (\%) & $1(6)$ & $5(38)$ & 0.06 \\
\hline
\end{tabular}

nocompromised, particularly through hematogenous seeding during periods of sepsis. In a recent large retrospective series of ventricular access device infection in premature neonates; however, sepsis alone without meningitis was not found to be a risk factor for device infection. ${ }^{30}$ Second, HIV-positive cases of cryptococcal meningitis generally have high titers of cryptococcal capsular polysaccharide due to massive fungal infestation with absent host immune response. ${ }^{8,13}$ This high level of CSF polysaccharide may increase the risk of mechanical shunt obstruction, but has not been specifically reported in this population. Third, shunting of infected CSF to an otherwise uninfected peritoneum may theoretically allow for systemic progression of Cryptococcus. To our knowledge, no such cases have been reported.

In this series, CSF shunting was overall well tolerated. Despite immunodeficiency, there were no cases of shunt infection. There were no cases of cryptococcal spread to the peritoneum. Two patients required repeat surgery for shunt revision. One patient developed an over-shunting subdural collection that was addressed definitively with shunt-valve revision. The second patient was found to have an obstructed shunt that was successfully explanted with no further need for CSF diversion. Four of the 5 deaths in patients who received shunts were secondary to severe medical comorbidities and terminal progression of immunodeficiency. It is unclear what role intracranial hyperten- sion played in the remaining death because that patient refused further workup.

It is our preference to offer shunt placement in patients with inadequate relief of intracranial hypertension symptoms after at least 3 lumbar punctures and initiation of appropriate antifungal therapy. Additionally, whether due to mycological relapse or to IRIS, patients with recurrent episodes of symptomatic elevated intracranial pressure may benefit from the sustained relief offered by shunting. As highlighted by previous authors, shunting during active fungal infection is not an issue if antifungal therapy has been started prior to implantation. ${ }^{25,26}$

IRIS is a clinical diagnosis representing exuberant tissue inflammation in patients experiencing rapid improvement in cellular immunity after initiation of HAART. CSF cultures are generally negative. In a recent prospective series, $22.5 \%$ of treated patients with cryptococcal meningitis developed IRIS within 12 weeks of starting HAART? Cerebral edema can be severe and associated with intracranial hypertension. ${ }^{41}$ Current infectious disease guidelines advocate for the use of corticosteroids such as prednisone to control the inflammatory response. ${ }^{26}$ Shunting when corticosteroids prove insufficient is not well described but was useful in this series.

Although we have placed lumboperitoneal shunts during our early experience with this problem, as well as in the management of pseudotumor cerebri due to the lack 


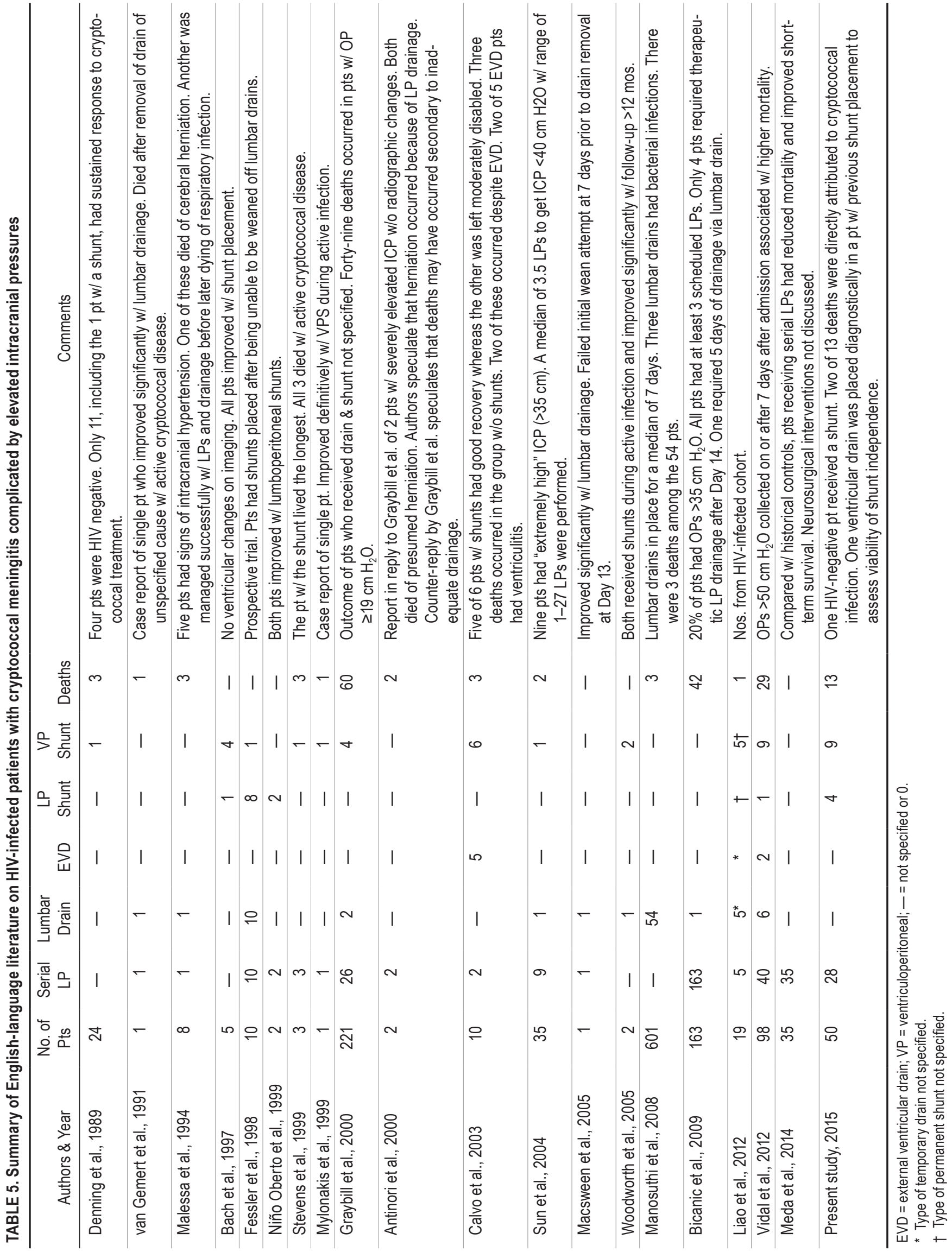


of ventriculomegaly, we now favor ventriculoperitoneal shunts exclusively. Lumboperitoneal shunts are often more technically difficult to place, particularly in obese patients. They are also more challenging to assess for failure because they cannot be easily tapped or palpated at the bedside. Even in cases where the ventricles are small, we have had good success using image navigation systems to place ventriculoperitoneal shunts, negating some of the purported technical advantages of lumbar shunt placement. Other authors have echoed similar difficulties with lumboperitoneal shunts..$^{40}$ Additionally, although the number of patients is too small for definitive assertion, patients suffering from IRIS seem to have better symptomatic relief with ventriculoperitoneal shunts than with lumboperitoneal shunts. More experience is needed to confirm this conjecture.

\section{Impact of Sex}

Of the 7 women in this series of 50 patients, 5 ultimately received shunts after serial lumbar puncture drainage failed, and 2 were treated with medical management only. It is not clear why female patients were more likely to receive shunts. There were no statistical differences in cryptococcal antigen levels or opening pressures based on sex. Perhaps there are similarities here with the pathophysiology of pseudotumor cerebri that reflect sex-specific responses to intracranial hypertension. Some cryptococcal meningitis series have demonstrated male sex as an overall negative prognostic factor, ${ }^{3}$ but to our knowledge none have correlated sex with need for CSF diversion.

\section{Role of Lumbar and Ventricular Drains}

In cases where clinical response to serial lumbar punctures is insufficient, we favor shunt placement over trials of external lumbar or ventricular drains. External drains generally require immobilization and demand a higher level of nursing comfort level because the open drain must be actively managed and monitored. In a large series by Manosuthi et al., ${ }^{18}$ lumbar drains were in place for a median of 7 days. In comparison, recovery from shunt placement is generally on the order of a few days and requires only routine postoperative care. Additionally, in the series by Fessler et al., ${ }^{12}$ there was an $80 \%$ rate of conversion to implanted shunts, bringing into question the durability of external lumbar drainage to offer sustained relief.

Infectious considerations are also particularly important in this population afflicted by immunodeficiency. In the cryptococcal meningitis series by Calvo et al., ${ }^{5}$ there was a $40 \%$ rate of ventriculitis in patients with external ventricular drains. Lumbar and ventricular drains are generally placed at the bedside and do not achieve the same standards of antisepsis required by intraoperative placement of shunts.

\section{Conclusions}

In cases where opening pressure is high, serial lumbar punctures combined with antifungal therapy are an effective first step in controlling intracranial hypertension in patients with cryptococcal meningitis. When clinical response is insufficient, however, ventriculoperitoneal shunt- ing is an effective next step. To our knowledge, this is the largest series on shunting in primarily HIV-positive patients with cryptococcal meningitis. We speculate that this may partly reflect a higher rate of medication noncompliance and subsequent readmissions for relapse.

In this series, nearly $30 \%$ of patients with cryptococcal meningitis underwent long-term CSF diversion. All patients in this series who were readmitted with mycological relapse underwent or were offered shunt placement. Patients who underwent serial lumbar punctures and then had shunts placed were statistically more likely to have had a pattern of increasing CSF cryptococcal antigen levels than patients who had serial lumbar punctures alone. After shunt insertion, patients generally had sustained relief from symptoms of intracranial hypertension, particularly headache. Ventricular shunts are favored over other methods of permanent or temporary CSF drainage.

\section{References}

1. Antinori S, Ridolfo AL, Gianelli E, Piazza M, Gervasoni C, Monforte AA: The role of lumbar puncture in the management of elevated intracranial pressure in patients with AIDS-associated cryptococcal meningitis. Clin Infect Dis 31:1309-1311, 2000

2. Bach MC, Tally PW, Godofsky EW: Use of cerebrospinal fluid shunts in patients having acquired immunodeficiency syndrome with cryptococcal meningitis and uncontrollable intracranial hypertension. Neurosurgery 41:1280-1283, 1997

3. Baldassarre R, Mdodo R, Omonge E, Jaoko W, Baddley J, Pappas P, et al: Mortality after clinical management of AIDS-associated cryptococcal meningitis in Kenya. East Afr Med J 91:145-151, 2014

4. Bicanic T, Brouwer AE, Meintjes G, Rebe K, Limmathurotsakul D, Chierakul W, et al: Relationship of cerebrospinal fluid pressure, fungal burden and outcome in patients with cryptococcal meningitis undergoing serial lumbar punctures. AIDS 23:701-706, 2009

5. Calvo A, Hernández P, Spagnuolo E, Johnston E: Surgical treatment of intracranial hypertension in encephalic cryptococcosis. Br J Neurosurg 17:450-455, 2003

6. Chan KH, Mann KS, Yue CP: Neurosurgical aspects of cerebral cryptococcosis. Neurosurgery 25:44-48, 1989

7. da Cunha Colombo ER, Mora DJ, Silva-Vergara ML: Immune reconstitution inflammatory syndrome (IRIS) associated with Cryptococcus neoformans infection in AIDS patients. Mycoses 54:e178-e182, 2011

8. Denning DW, Armstrong RW, Lewis BH, Stevens DA: Elevated cerebrospinal fluid pressures in patients with cryptococcal meningitis and acquired immunodeficiency syndrome. Am J Med 91:267-272, 1991

9. Denning DW, Tucker RM, Hanson LH, Hamilton JR, Stevens DA: Itraconazole therapy for cryptococcal meningitis and cryptococcosis. Arch Intern Med 149:2301-2308, 1989

10. De Wytt CN, Dickson PL, Holt GW: Cryptococcal meningitis. A review of 32 years experience. J Neurol Sci 53:283292, 1982

11. Diamond RD, Bennett JE: Prognostic factors in cryptococcal meningitis. A study in 111 cases. Ann Intern Med 80:176181,1974

12. Fessler RD, Sobel J, Guyot L, Crane L, Vazquez J, Szuba MJ, et al: Management of elevated intracranial pressure in patients with Cryptococcal meningitis. J Acquir Immune Defic Syndr Hum Retrovirol 17:137-142, 1998

13. Graybill JR, Sobel J, Saag M, van Der Horst C, Powderly W, Cloud G, et al: Diagnosis and management of increased 
intracranial pressure in patients with AIDS and cryptococcal meningitis. Clin Infect Dis 30:47-54, 2000

14. Liao CH, Chi CY, Wang YJ, Tseng SW, Chou CH, Ho CM, et al: Different presentations and outcomes between HIVinfected and HIV-uninfected patients with Cryptococcal meningitis. J Microbiol Immunol Infect 45:296-304, 2012

15. Liliang PC, Liang CL, Chang WN, Chen HJ, Su TM, Lu K, et al: Shunt surgery for hydrocephalus complicating cryptococcal meningitis in human immunodeficiency virus-negative patients. Clin Infect Dis 37:673-678, 2003

16. Macsween KF, Bicanic T, Brouwer AE, Marsh H, Macallan DC, Harrison TS: Lumbar drainage for control of raised cerebrospinal fluid pressure in cryptococcal meningitis: case report and review. J Infect 51:e221-e224, 2005

17. Malessa R, Krams M, Hengge U, Weiller C, Reinhardt V, Volbracht L, et al: Elevation of intracranial pressure in acute AIDS-related cryptococcal meningitis. Clin Investig 72:1020-1026, 1994

18. Manosuthi W, Sungkanuparph S, Chottanapund S, Tansuphaswadikul S, Chimsuntorn S, Limpanadusadee P, et al: Temporary external lumbar drainage for reducing elevated intracranial pressure in HIV-infected patients with cryptococcal meningitis. Int J STD AIDS 19:268-271, 2008

19. McLone DG, Siqueira EB: Post-meningitic hydrocephalus and syringomyelia treated with a ventriculoperitoneal shunt. Surg Neurol 6:323-325, 1976

20. Meda J, Kalluvya S, Downs JA, Chofle AA, Seni J, Kidenya $B$, et al: Cryptococcal meningitis management in Tanzania with strict schedule of serial lumber punctures using intravenous tubing sets: an operational research study. J Acquir Immune Defic Syndr 66:e31-e36, 2014

21. Mylonakis E, Merriman NA, Rich JD, Flanigan TP, Walters BC, Tashima KT, et al: Use of cerebrospinal fluid shunt for the management of elevated intracranial pressure in a patient with active AIDS-related cryptococcal meningitis. Diagn Microbiol Infect Dis 34:111-114, 1999

22. Newton PN, Thai H, Tip NQ, Short JM, Chierakul W, Rajanuwong A, et al: A randomized, double-blind, placebocontrolled trial of acetazolamide for the treatment of elevated intracranial pressure in cryptococcal meningitis. Clin Infect Dis 35:769-772, 2002

23. Niño Oberto S, Estañol Vidal B, García Ramos G, Vega Boada F, Sierra Madero J: [Significance of intracranial hypertension management in cryptococcal meningitis in patients with acquired immunodeficiency syndrome. Report of 2 cases.] Rev Investig Clín 51:303-307, 1999 (Span)

24. Pappas PG: Managing cryptococcal meningitis is about handling the pressure. Clin Infect Dis 40:480-482, 2005

25. Park MK, Hospenthal DR, Bennett JE: Treatment of hydrocephalus secondary to cryptococcal meningitis by use of shunting. Clin Infect Dis 28:629-633, 1999

26. Perfect JR, Dismukes WE, Dromer F, Goldman DL, Graybill JR, Hamill RJ, et al: Clinical practice guidelines for the management of cryptococcal disease: 2010 update by the Infectious Diseases Society of America. Clin Infect Dis 50:291-322, 2010

27. Richardson PM, Mohandas A, Arumugasamy N: Cerebral cryptococcosis in Malaysia. J Neurol Neurosurg Psychiatry 39:330-337, 1976

28. Schoeman JF, Honey EM, Loock DB: Raised ICP in a child with cryptococcal meningitis: CT evidence of a distal CSF block. Childs Nerv Syst 12:568-571, 1996

29. Shoham S, Cover C, Donegan N, Fulnecky E, Kumar P: Cryptococcus neoformans meningitis at 2 hospitals in Washington, D.C.: adherence of health care providers to published practice guidelines for the management of cryptococcal disease. Clin Infect Dis 40:477-479, 2005

30. Spader HS, Hertzler DA, Kestle JRW, Riva-Cambrin J: Risk factors for infection and the effect of an institutional shunt protocol on the incidence of ventricular access device infections in preterm infants. J Neurosurg Pediatr 15:156-160, 2015

31. Stevens DA, Denning DW, Shatsky S, Armstrong RW, Adler JD, Lewis BH: Cryptococcal meningitis in the immunocompromised host: intracranial hypertension and other complications. Mycopathologia 146:1-8, 1999

32. Sun HY, Hung CC, Chang SC: Management of cryptococcal meningitis with extremely high intracranial pressure in HIVinfected patients. Clin Infect Dis 38:1790-1792, 2004

33. Tan CT: Intracranial hypertension causing visual failure in cryptococcus meningitis. J Neurol Neurosurg Psychiatry 51:944-946, 1988

34. Tan CT, Kuan BB: Cryptococcus meningitis, clinical-CT scan considerations. Neuroradiology 29:43-46, 1987

35. Tang LM: Ventriculoperitoneal shunt in cryptococcal meningitis with hydrocephalus. Surg Neurol 33:314-319, 1990

36. Tay CH, Chew WL, Lim LC: Cryptococcal meningitis: its apparent increased incidence in the Far East. Brain 95:825832,1972

37. Tjia TL, Yeow YK, Tan CB: Cryptococcal meningitis. J Neurol Neurosurg Psychiatry 48:853-858, 1985

38. van Gemert HM, Vermeulen M: Treatment of impaired consciousness with lumbar punctures in a patient with cryptococcal meningitis and AIDS. Clin Neurol Neurosurg 93:257-258, 1991

39. Vidal JE, Gerhardt J, Peixoto de Miranda ÉJ, Dauar RF, Oliveira Filho GS, Penalva de Oliveira AC, et al: Role of quantitative CSF microscopy to predict culture status and outcome in HIV-associated cryptococcal meningitis in a Brazilian cohort. Diagn Microbiol Infect Dis 73:68-73, 2012

40. Woodworth GF, McGirt MJ, Williams MA, Rigamonti D: The use of ventriculoperitoneal shunts for uncontrollable intracranial hypertension without ventriculomegally secondary to HIV-associated cryptococcal meningitis. Surg Neurol 63:529-532, 2005

41. York J, Bodi I, Reeves I, Riordan-Eva P, Easterbrook PJ: Raised intracranial pressure complicating cryptococcal meningitis: immune reconstitution inflammatory syndrome or recurrent cryptococcal disease? J Infect 51:165-171, 2005

\section{Disclosure}

The authors report no conflict of interest concerning the materials or methods used in this study or the findings specified in this paper.

\section{Author Contributions}

Conception and design: Gopinath, Cherian. Acquisition of data: Cherian. Analysis and interpretation of data: Cherian. Drafting the article: Cherian. Critically revising the article: all authors. Reviewed submitted version of manuscript: all authors. Approved the final version of the manuscript on behalf of all authors: Gopinath. Statistical analysis: Cherian. Administrative/technical/material support: Gopinath. Study supervision: Gopinath.

\section{Correspondence}

Shankar P. Gopinath, Department of Neurosurgery, Baylor College of Medicine, 6501 Fannin St., Ste. NC100, Houston, TX 77030.email: shankarg@bcm.edu. 